\title{
Iris metastasis: an unusual cause of anterior uveitis
}

\author{
CH Chua, W Westlake, w Rich
}

\section{Summary \\ We report a case of anterior uveitis due to iris metastasis in a patient with known carcinomatosis.}

Keywords: iris metastasis, anterior uveitis

In a patient with a known history of cancer, pain in and around the eye should arouse the suspicion of iris metastasis. The causes of such pain include anterior uveitis and sometimes secondary severe glaucoma. Treatment is usually effective in alleviating the symptoms.

\section{Case report}

A 47-year-old woman developed multiple nodular lesions on her back, associated with generalised malaise. She was a non-smoker and had no previous history of note. Further examination revealed smaller lesions on her neck and a $1-\mathrm{cm}$ breast lump that was clinically benign. Biopsy of the back lesion revealed subcutaneous fatty tissue with anaplastic cell infiltration. Histology suggested a neuroendocrine oat cell carcinomatous origin. Computed tomography (CT) of the abdomen showed multiple low-attenuation areas present throughout both lobes of the liver, consistent with hepatic secondaries. Other organs were clear. CT scan of the thorax was normal as was the skeletal isotope scan.

In view of the widespread metastasis, further extensive search for the primary was not thought to be helpful. The patient was informed of the diagnosis and she agreed to undergo a trial of chemotherapy consisting of cisplatin, adriamycin and vindesine in combination repeated at four-weekly intervals. Her life expectancy was estimated to be 6-12 months.

After two months of chemotherapy, she developed a constant dull ache over her left eye. The pain persisted for about five weeks before she sought medical advice. When seen by the ophthalmologist, her pain had disappeared for four days. Her visual acuity was normal in both eyes but slit-lamp examination revealed a whitish lesion situated at the iris root at 5 o'clock (figure 1). There were keratitic precipitates on the endothelium with flare, but no anterior chamber inflammation. Her intraocular pressure was $18 \mathrm{mmHg}$ in both eyes. Fundal examination did not show any choroidal metastasis. The eye pain was attributed to a preceding attack of anterior uveitis secondary to the tumour. As the eye was quiet, no intervention was deemed necessary.

The chemotherapy was continued but the size of the lesions on her back and neck remained unchanged. However, during the six-month ophthalmic follow-up the iris mass progressively enlarged and became vascularised (figure 2). Ultrasound of the eye revealed choroidal deposits (figure 3 ). The visual acuity and intraocular pressure remained normal and there was no recurrence of the ocular pain.

In view of her continued deteroriating health and absence of any ocular symptoms, no intervention was considered necessary.

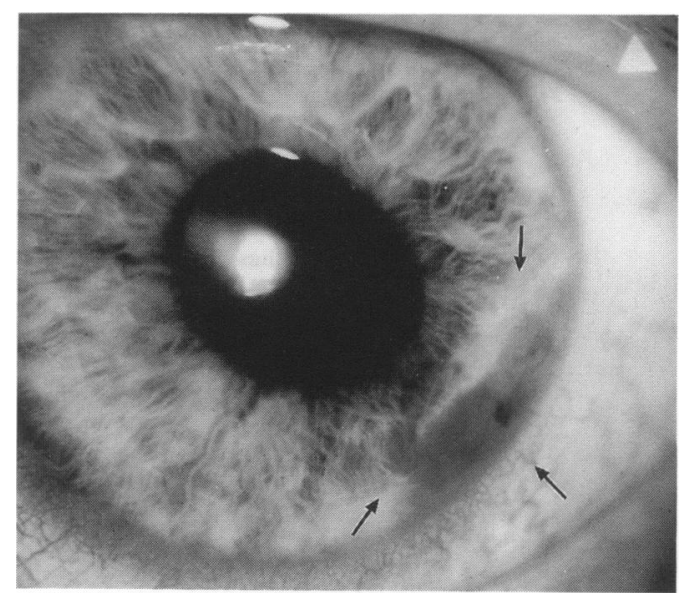

Figure 1 Arrows indicating the site of iris metastasis

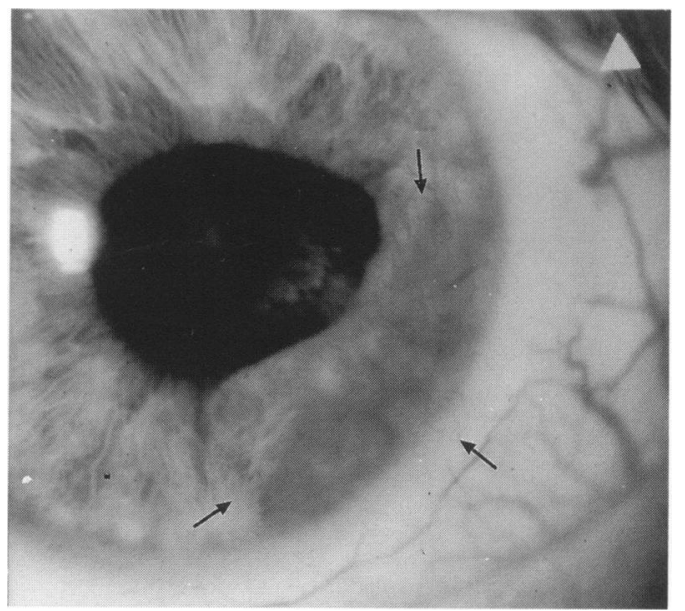

Figure 2 Arrows indicating the iris metastasis which had increased in size 


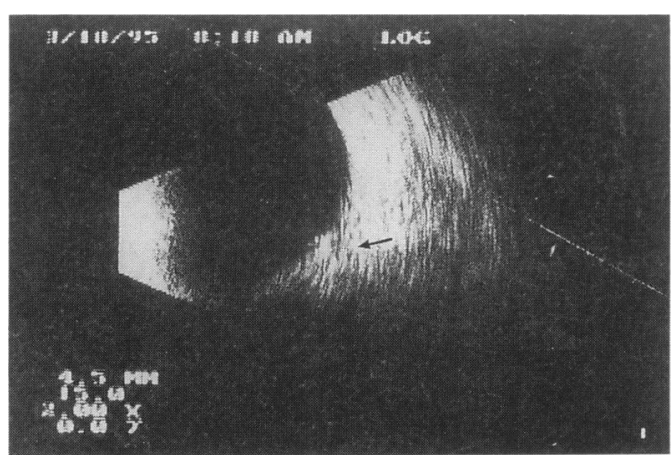

Figure 3 Arrow indicating choroidal deposit on ultrasound scan

\begin{tabular}{|l|}
\hline Iris metastasis: features \\
\hline - less frequent than posterior uveal metastases \\
- most common primaries are breast, lung and \\
gastrointestinal carcinoma \\
- lightly pigmented in most cases \\
- can be located in any quadrant of the iris \\
- presents with anterior chamber reaction such \\
- as anterior uveitis or secondary glaucoma \\
treatment is palliative with topical medication \\
or radiotherapy \\
prognosis is poor in patients with iris \\
metastases
\end{tabular}

\section{Discussion}

Iris metastases are uncommon. ${ }^{1}$ The most common sources are lung, breast and gastrointestinal carcinoma. ${ }^{2}$ Most iris metastases occur in patients with known carcinoma, though in $25 \%$ of cases these may be the first manifestation of carcinomatosis. ${ }^{3}$ The tumours usually present with anterior segment reactions. The most common reactions are inflammation in $44 \%,{ }^{4}$ glaucoma $7 \%$ and hyphaema. It is important to consider iris

1 Ferry A, Font R. Carcinoma metastatic to the eye and orbit. II. Arch Ophthalmol 1975; 93: 472-82.

2 Shields JA. Diagnosis and management of intraocular tumors. St Louis: Mosby, 1983; pp 278-321.

3 Sabbagh R, Shields CL, Shields JA, Ehya J, Finegan JT. Spontaneous hyphema: initial manifestation of lung carcinoma. $¥ A M A$ 1991; 266: 3194.

\section{Learning points}

- iris metastasis is a rare cause of anterior uveitis

- iris metastasis should be suspected in cancer patients with ocular pain

- ocular ultrasound can help confirm diagnosis by revealing deposits in the choroid or the orbit

- treatment is effective in reducing the morbidity

metastasis in the differential diagnosis of any iris lesions to avoid unnecessary surgical intervention. Unlike iris melanomas, which frequently involve inferior quadrant, metastases can involve any quadrant. They are typically yellowish in colour but can become pigmented from reactive stromal cell proliferation. Being friable, they can sometimes seed into the aqueous, producing pseudohypopyon.

The evaluation should include a comprehensive systemic review for other metastatic disease. Ultrasound and CT of the ocular structures can help diagnosis by revealing deposits in the choroid and the orbit. Other helpful tests include fine needle aspiration biopsy in uncertain cases. Serum carcinoembryonic antigen can also be useful as it is raised $(>10 \mathrm{ng} / \mathrm{ml})$ in tumours of endodermal origin but normal in patients with uveal melanoma. ${ }^{6}$

Therapy is aimed at alleviating ocular symptoms. The indications are pain, uveitis, or glaucoma. Topical steroid and anti-glaucoma treatment are useful. The tumour may undergo regression with chemotherapy for the primary tumour. In unresponsive cases, radiation therapy using 3000 to $4000 \mathrm{cGy}$ over a three- to five-week period achieves a success rate of over $80 \%{ }^{7}$ The long-term survival is poor in patients with ocular secondaries, averaging 7.4 to 10.5 months. ${ }^{8}$ This report illustrates the importance of suspecting iris metastasis in patients with cancer and a painful eye.

4 Spencer WH (ed). Ophthalmic pathology: uveal tract tumor Philadelphia, Pa: WB Saunders Co, 1986; pp 1575-87.

5 Ferry AP, Font RL. Carcinoma metastatic to the eye and orbit. II. Arch Ophthalmol 1974; 92: 276-86.

6 Denslow GT, Kieler RA. Metastatic adenocarcinoma to the anterior uvea and increased carcinoembryonic antigen levels. Am $\mathcal{J}$ Ophthalmol 1978; 85: 363-7. 